\title{
Walking after incomplete spinal cord injury with an implanted neuromuscular electrical stimulation system and a hinged knee replacement: a single-subject study
}

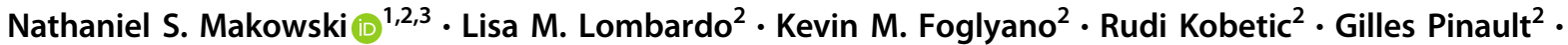 \\ Stephen M. Selkirk ${ }^{2,3} \cdot$ Ronald J. Triolo ${ }^{2,3}$
}

Received: 11 May 2020 / Revised: 27 August 2020 / Accepted: 31 August 2020

This is a U.S. government work and not under copyright protection in the U.S.; foreign copyright protection may apply 2020

\begin{abstract}
Study design Single-subject repeated measures study.

Objectives Neuromuscular electrical stimulation (NMES) can enhance walking for people with partial paralysis from incomplete spinal cord injury (iSCI). This single-subject study documents an individual's experience who both received an experimental implanted NMES system and underwent clinical bilateral hinged total knee arthroplasty (TKA). She walked in the community with knee pain prior to either intervention. Walking performance improved with an implanted NMES system. Knee pain and instability continued to worsen over time and eventually required TKA. This study evaluates the effects of these interventions.

Setting Louis Stokes Cleveland Veterans Affairs Medical Center, Cleveland OH, USA.

Methods The differential and combined effects of NMES and hinged knee replacement were assessed in terms of walking speed, toe clearance, knee angle, and participant perceptions with and without stimulation assistance both before and after TKA.

Results The combined approach both reduced pain and restored walking ability to levels achieved prior to developing significant knee pain that prevented walking without NMES. There was an interaction effect between NMES and TKA on walking speed. Toe clearance consistently improved with stimulation assistance and TKA prevented significant knee hyperextension. The greatest impact was on endurance. Knee replacement re-enabled long distance walking with the addition of stimulation again more than doubling her maximum walking distance from 214 to $513 \mathrm{~m}$.

Conclusions These data support further research of combined implantable interventions that may benefit people with iSCI. Furthermore, joint laxity and pain may not necessarily be contraindications to NMES if addressed with conventional clinical treatments.
\end{abstract}

Supplementary information The online version of this article (https:// doi.org/10.1038/s41394-020-00336-8) contains supplementary material, which is available to authorized users.

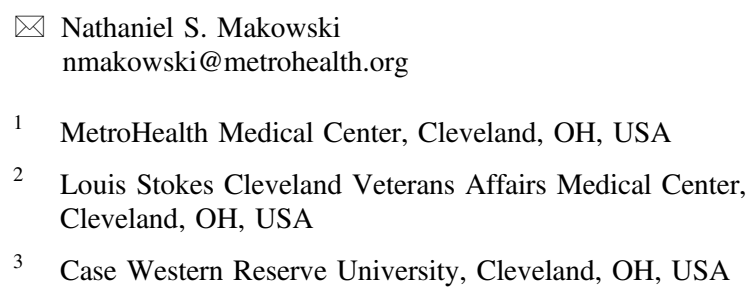

3 Case Western Reserve University, Cleveland, OH, USA

\section{Introduction}

In the United States, $\sim 67 \%$ of all spinal cord injuries are incomplete (iSCI) [1]. This is partly due to advances in acute medical management, research, and rehabilitation. People with iSCI maintain some level of function but require additional assistance (e.g., wheelchair, crutches) for community access. Walking performance after iSCI can be improved by using neuromuscular electrical stimulation (NMES) to generate the muscle forces necessary to assist ambulation [2-5]. People with SCI are also living longer, and are now confronted with degenerative changes due to aging, such as arthrosis. Spasticity and muscular imbalances after partial paralysis can further increase the strain on weight bearing joints such as the hips and knees. Joint 
replacement is not commonly associated with individuals with SCI, but may be an appropriate intervention for household or community ambulators who develop pain or other joint injuries that limit function [6, 7]. Combined interventions to improve or maintain ambulatory function, such as integrating implanted NMES with total joint replacements, are rare since there are currently no commercially available implanted NMES systems for walking. As interventions are developed, combined approaches may become more common. This report describes the case of an individual with iSCI whose walking improved with assistance from an experimental implanted NMES system, but deteriorated over time due to knee pain and joint laxity. Pain and laxity were addressed with clinically prescribed bilateral knee replacement, which allowed resumption of the prior benefits of NMES assistance. The differential contributions of joint replacement and NMES are documented as they enabled return to peak ambulatory performance.

\section{Participant description}

The study participant was a 56-year-old female injured in 1983 resulting in a C6 AIS C SCI with diminished sensation, intact proprioception, and weakness throughout the lower limb (Table 1). The subsequent progression of events is shown in Fig. 1. After her iSCI, she was a limited community ambulator and primarily walked with forearm crutches, and supplemented this mobility with a manual wheelchair. After years of walking with decreased muscle strength and imbalance, the ligaments supporting her knee joint were stretched and she walked with significant knee hyperextension to maintain stability in stance. Hyperextending allowed her to walk with less conscious effort to control her knees. She attempted to use ankle and knee orthoses to prevent excessive knee range of motion but found them uncomfortable to the point of being intolerable. Thirty years post iSCI she received a surgically implanted 8-channel NMES system to facilitate ambulation as described previously, and has walked with the system for

Table 1 Lower extremity manual muscle test scores 3 months after TKA.

\begin{tabular}{lll}
\hline & Left side & Right side \\
\hline Hip flexion & $1+$ & $2+$ \\
Hip extension & $2+$ & 3 \\
Hip abduction & 1 & 1 \\
Hip adduction & 2 & 2 \\
Knee extension & $3+$ & 3 \\
Knee flexion & 0 & 1 \\
Ankle dorsiflexion & 0 & 1 \\
Ankle plantarflexion & 4 & $3+$ \\
\hline
\end{tabular}

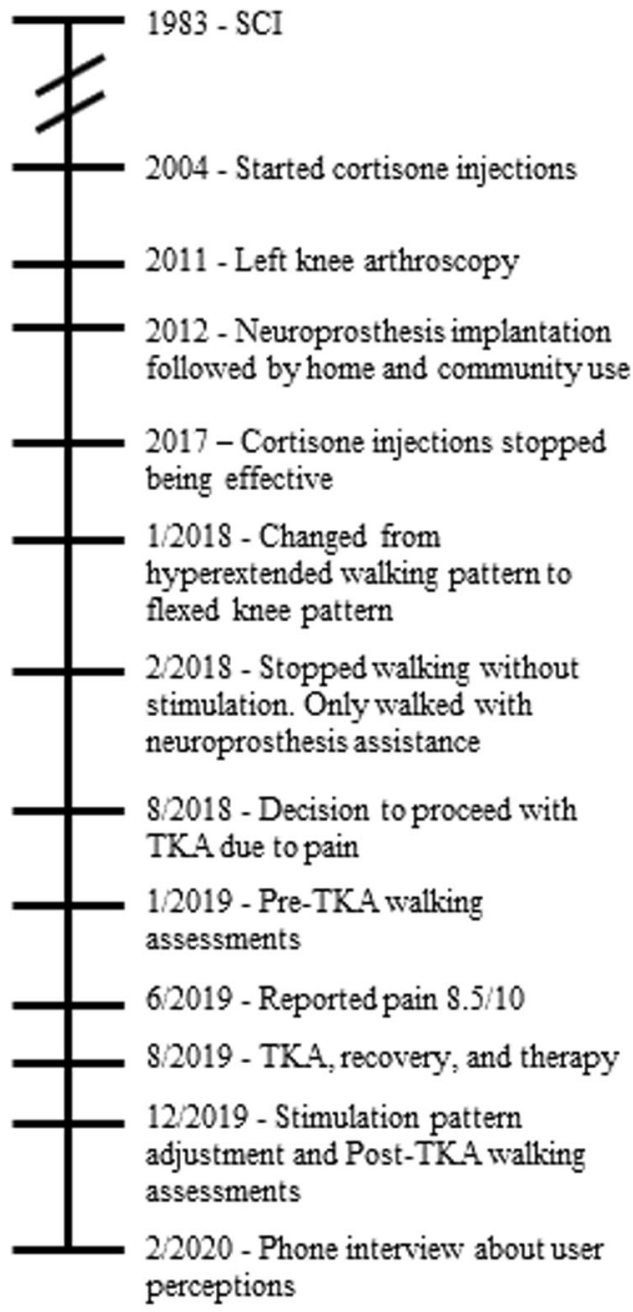

Fig. 1 Timeline showing the sequence of injury, pain development, interventions, and assessments. Periods of time are not to scale.

the past 6 years $[8,9]$. The implanted neuroprosthesis targeted nerves to contract the following muscles bilaterally to assist hip and knee flexion and ankle dorsiflexion [10]: tensor fasciae latae, sartorius, short head of biceps femoris (SHBF), and tibialis anterior. Stimulation timing and pulse duration for each muscle were programmed to respond to wireless sensor inputs transmitted to an external controller. Steps were triggered by contralateral crutch strike through wireless accelerometers mounted on her forearm crutches [11]. The implanted system enabled her to continue walking beyond what could have been achieved with voluntary effort alone by decreasing the strain on her upper extremities that was required to bring her feet forward with each step without stimulation. At short distances her walking speed remained relatively consistent $(\sim 0.2 \mathrm{~m} / \mathrm{s})$ with and without stimulation assistance. The greatest impact of NMES was in walking long distances; maximum walk distance increased over $400 \%$ (393 m improvement) at a steady walking speed $(0.22 \mathrm{~m} / \mathrm{s})$, which was not sustainable 
without stimulation. She used NMES regularly to walk in the community.

Her knee pain progressively worsened and compromised volitional walking; she became reliant on the neuroprosthesis for all ambulatory tasks. She altered her gait pattern to prevent knee pain caused by hyperextension, which resulted in slower walking and required significantly more concentration and voluntary effort. Knee pain continued to progress; she reported pain at 8.5 on a 10 -point numeric rating scale prior to scheduling joint replacement surgery. Knee hyperextension was greater than $20^{\circ}$ and flexion was $120^{\circ}$ with valgus alignment. On August 23, 2019, she underwent bilateral total knee arthroplasty (TKA) using rotating hinged knee joints. This type of hinged joint is not as common as traditional TKA, but can be useful in complex cases with significant instability and inadequate collateral ligaments to support the joint [12]. The TKA prescription, device selection, and immediate pre- and postoperative evaluations were completed as part of routine clinical care without involvement of the NMES research team.

Knee replacement surgery did not disrupt the leads that crossed the knees to stimulate tibialis anterior and kept the NMES system intact and fully operational. During the recovery process she exercised with her implanted NMES system to regain muscle strength, endurance, and cardiovascular conditioning. Eleven weeks after TKA, she regained sufficient function to begin gait training in physical therapy. Physical therapy included practicing walking both with and without stimulation. At her follow-up appointment on September 16, 2019, her pain was 5/10 and range of motion was within normal limits while preventing excessive knee hyperextension.

This single-subject repeated measures study evaluated the differential and combined effects of TKA on her voluntary and NMES assisted walking as she regained and surpassed her peak performance originally exhibited with NMES alone.

\section{Methods}

Data were collected with and without stimulation assistance at the following time points and compared to peak performance after first receiving the NMES system: (1) after developing intolerable knee pain 7 months prior to TKA surgery, and (2) after completing therapy post-TKA. Ambulatory performance was assessed in terms of: (1) walking speed via the $10 \mathrm{~m}$ walk test, (2) walking distance via the 6 min Walk Test (6MWT), (3) maximum walking time and distance, (4) peak toe clearance during swing and peak knee extension during stance, and (5) user experience and perceptions through a semistructured interview. The stimulation pattern was adjusted to optimize performance at each time point. Unrelated to the knee replacement, the lead wires to electrodes targeting SHBF and sartorius on the right side developed discontinuities and were nonfunctional after she developed significant knee pain, so they were not included in the stimulation patterns applied during the follow-up assessments.

The $10 \mathrm{~m}$ walk test determined peak walking speed over a short distance. Five repetitions were completed with and without stimulation at each session, separated by $10 \mathrm{~min}$ of rest to limit fatigue. The 6MWT evaluated walking speed over a duration that is more representative of activities of daily living. Two repetitions were completed for each condition at each time point with $1 \mathrm{~h}$ of rest between trials. A single maximum walk test was completed with and without stimulation due to the physical demands on the subject and the significant time commitment required. Distance and number of steps were collected at $2 \mathrm{~min}$ intervals. There was a 2.5 -h rest period between the two maximum walk trials. The Borg Rating of Perceived Exertion (6-20 point scale) was collected after the 6MWT and maximum walk test. The testing sequence was randomized for trials with and without stimulation.

Kinematic data were collected with a Vicon Vantage motion capture system during the $10 \mathrm{~m}$ walk tests. Reflective markers were placed on anatomical landmarks based on the Helen Hayes marker set [13]. Joint angles were calculated using the Vicon Plug-in-Gait biomechanical model. Reported outcomes are peak knee extension in stance and toe clearance during swing because the knee arthroplasty directly impacted knee extension range, and stimulation addressed the toe drag characteristic of stepping without stimulation. Steps where she hyperextended and did not maintain a flexed knee posture prior to TKA were not included in the analysis. Toe clearance was measured as the rise in height of the second metatarsal marker after swing initiation. Each gait cycle created one sample for each outcome. There were at least 60 repetitions for each condition. A separate trial with instrumented forearm crutches determined changes in peak upper extremity loading within steps with and without NMES.

Descriptive statistics including means, standard deviations, and ranges are presented due to the limited number of samples for some outcomes. Analysis of variance was used to detect changes $(p<0.05)$ in the $10 \mathrm{~m}$ walking speed and kinematic outcomes before and after TKA while walking with and without stimulation assistance. Post-hoc comparisons determined differences between conditions. A twosample $t$-test compared forearm loading after TKA with and without stimulation assistance.

The participant completed a semistructured interview after using the system at home and in the community for two and a half months after knee replacement. Questions 
focused on her perceptions of the interventions' effects, considerations about the surgeries, and perspectives on what she would do differently.

We certify that all applicable institutional and governmental regulations concerning the ethical use of human volunteers were followed during the course of this research. The registry number is NCT01570816.

\section{Results}

The participant walked after recovering from knee surgery without any adverse effects on any components of her implanted neuroprosthesis. NMES improved toe clearance and the TKA prevented extreme hyperextension. Still-images from videos taken with and without stimulation assistance both before and after surgery are shown in Fig. 2. Supplementary videos show the walking pattern in each condition.

\section{Walking speed}

Walking speed slowed by almost $40 \%$ both with and without stimulation as knee pain developed and then returned to prior levels after knee replacement surgery (Table 2). There was an interaction effect between TKA and stimulation $(p<0.01)$. Prior to knee surgery, peak walking speed $(10 \mathrm{~m}$ walk test) was $50 \%$ faster with stimulation. After surgery walking speeds increased overall by $80 \%$, but peak speeds were $25 \%$ faster without stimulation assistance. These speeds were comparable to her prepain performance with $10 \mathrm{~m}$ gait speeds of $\sim 0.2 \mathrm{~m} / \mathrm{s}$ with or without stimulation. The 6MWT and maximum distance walk were not completed in the session prior to knee surgery because the participant experienced too much pain walking without stimulation. These values were only compared after surgery. Gait speeds were comparable with and without stimulation during longer walks with average speeds of $\sim 0.2 \mathrm{~m} / \mathrm{s}$ during
Fig. 2 Stills from video showing improved toe clearance (swing) and knee extension (stance). The following conditions: a) without stimulation before TKA, b) with stimulation before TKA, c) without stimulation after TKA, and $\mathbf{d}$ ) with stimulation after TKA.
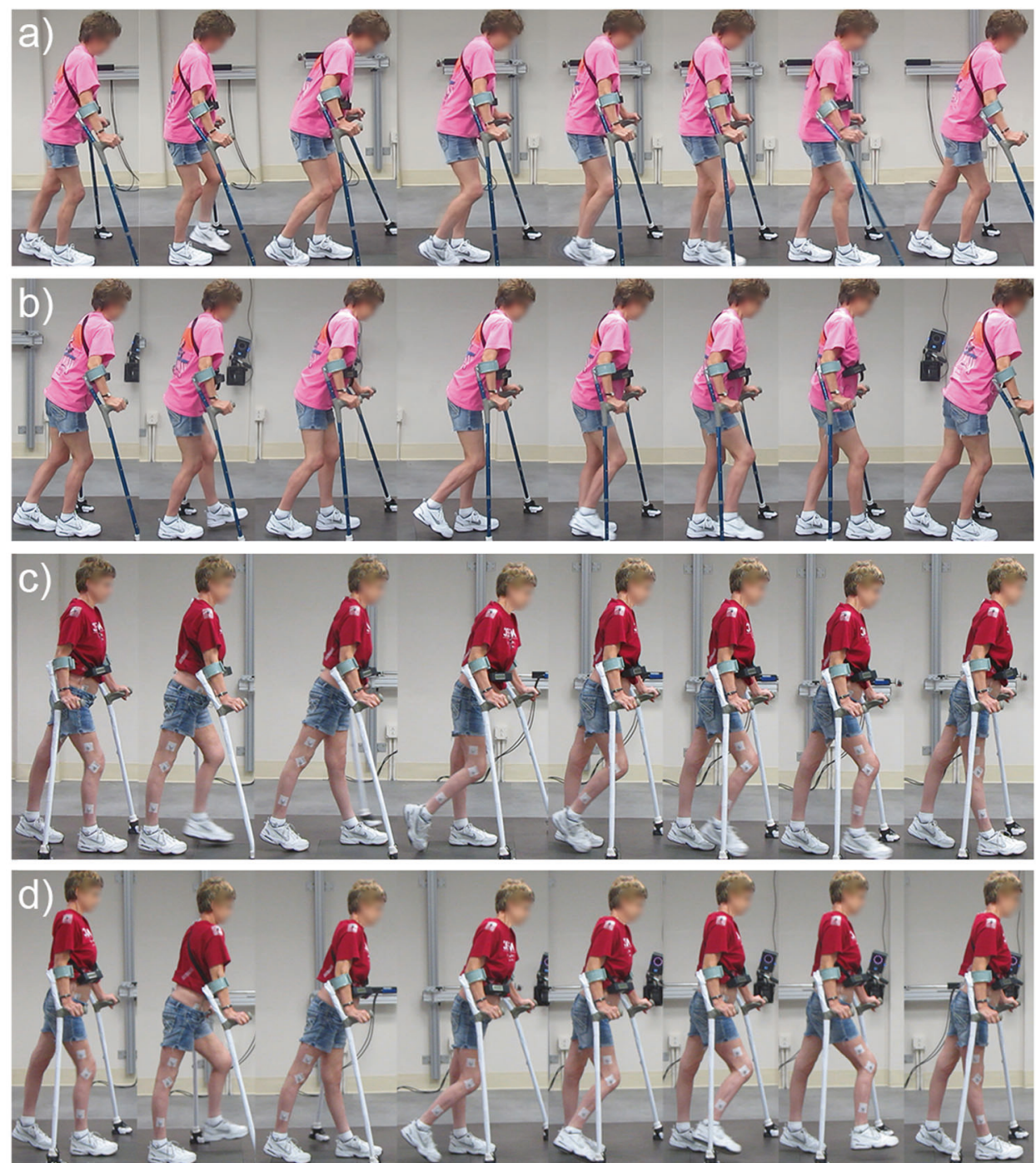
Table 2 Walking speeds $(\mathrm{m} / \mathrm{s})$ and perceived effort before and after joint replacement with and without stimulation assistance.

\begin{tabular}{lll}
\hline & Pre knee surgery & Post knee surgery \\
\hline 10 m walk-no stimulation & $0.10(0.00)$ & $0.25(0.03)$ \\
10 m walk -with stimulation & $0.15(0.02)$ & $0.20(0.00)$ \\
6 min walk test—no stimulation & N/A & $0.21(0.2-0.22)$ \\
6-min walk test—with stimulation & N/A & $0.19(0.18-0.19)$ \\
Borg RPE during 6 min walk test-no stimulation & N/A & 15.5 \\
Borg RPE during 6 min walk test-with stimulation & N/A & 13 \\
Max walk—no stimulation (21 min) & N/A & 0.16 \\
Max walk—with stimulation (49 min) & N/A & 0.17 \\
\hline
\end{tabular}

$10 \mathrm{~m}$ walk-mean (SD), 6 min walk test-mean (range).

N/A not available.

the $6 \mathrm{MWT}$ and $\sim 0.17 \mathrm{~m} / \mathrm{s}$ during the max walk. Average gait speed during the max walk was slightly faster (14\%) compared to her longest walk without stimulation and slightly slower $(23 \%)$ than her longest walk with stimulation before she developed significant knee pain. The participant reported less effort by 2.5 points while walking with stimulation assistance after the 6MWT.

Figure 3 shows the distance and average gait speed over each 2-min period during the maximum distance walk with and without stimulation after TKA. With stimulation, the participant maintained a steady walking speed, while without stimulation she started out walking faster during the first $4 \mathrm{~min}$ and then slowed down similar to her long distance walking prior to developing significant knee pain. With this change in pace, total distance was the same between the two conditions at the 10-min mark. She continued walking without stimulation while maintaining a slower speed. However, with stimulation she maintained the ability to continue walking beyond her stopping point with volitional effort alone. She walked an additional $27 \mathrm{~min}$ and about $300 \mathrm{~m}$ further with stimulation assistance, which were more than twice as long and as far as without the neuroprosthesis. These distances are 54 and 5\% greater than her longest walks prior to developing significant knee pain without $(139 \mathrm{~m})$ and with stimulation $(488 \mathrm{~m})$ [8]. She stopped walking without stimulation due to pain in her right knee and her quadriceps muscles felt like they were "burning"; she also reported pain in one of her wrists and one knee. She stopped walking with stimulation due to pain in her wrists, and had no pain in her knees. In both cases the greatest pain was in the right wrist. Wrist pain was similar at the end of the two walks. She reported similar fatigue and effort levels at the end of each trial despite walking significantly longer with stimulation.

\section{Kinematics and kinetics}

Toe clearance increased with stimulation assistance both before and after knee surgery. Average peak toe clearance
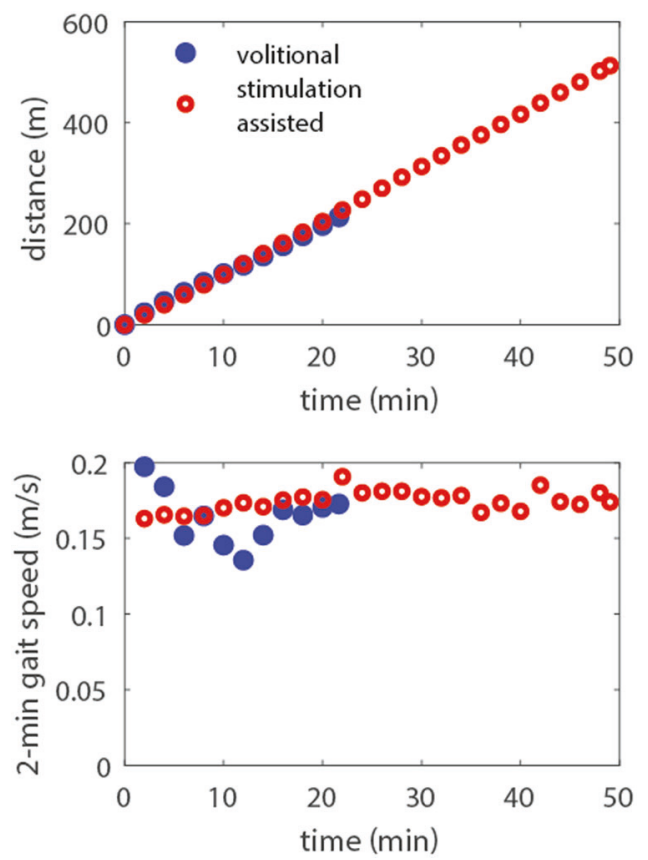

Fig. 3 Walking distance and speed during the maximum walk tests. Cumulative walking distance at 2-min increments (top) and gait speed at 2-min increments (bottom).

increased $4-5 \mathrm{~cm}$ with stimulation and exhibited an interaction effect $(p=0.03)$ with slightly greater toe clearance after surgery (Table 3). However, toe clearance was not significantly different without stimulation as she continued to drag her toes without NMES assistance. Stimulation facilitated toe clearance by dorsiflexing the ankle, whereas stepping without stimulation involved dragging the toes across the floor with each step. It is important to note that peak toe clearance without stimulation is above zero because the foot rotated as the tip of the shoe drags on the ground whereas stimulation maintained proper foot orientation and the toe successfully cleared the floor.

Table 3 shows the maximum extension angle achieved by both knees across the entire session. Prior to TKA, the participant was able to walk slowly with $\sim 12^{\circ}$ of flexion to 
Table 3 Toe clearance and knee extension kinematic measures as well as peak forearm forces measured by instrumented crutches.

\begin{tabular}{lll}
\hline & Pre knee surgery Mean (SD) & Post knee surgery Mean (SD) \\
\hline Peak toe clearance without stimulation assistance (mm) & $22(16)$ & $26(9)$ \\
Peak toe clearance with stimulation assistance (mm) & $63(16)$ & $78(33)$ \\
Peak knee extension during stance without stimulation assistance (mean) (degrees) & $-15(11)$ & $16(2)$ \\
Peak knee extension during stance with stimulation assistance (mean) (degrees) & $-12(10)$ & $17(2)$ \\
Maximum peak knee hyperextension (degrees) pre knee surgery value was during & 29 & 20 \\
unintended hyperextension & N/A & $145.8(5.2)$ \\
Peak forearm force during stance without stimulation assistance (N) & N/A & $123.5(7.1)$ \\
Peak forearm force during stance with stimulation assistance &
\end{tabular}

Positive knee extension values represent extension beyond neutral. Negative knee extension values represent knee flexion.

prevent the hyperextension that exacerbated pain. However, conscious attempts to prevent hyperextension prior to TKA were often unsuccessful and resulted in knee angles similar to those exhibited prior to developing pain $\left(\sim 30^{\circ}\right.$ past neutral) as evident in Table 3. After TKA surgery, the joint replacement mechanically limited her knee ranges of motion, and she could safely extend to neutral $(p<0.01)$ without actively maintaining flexion or undue concerns of pain. Stimulation did not significantly affect stance phase knee extension $(p=0.06)$ as the hard stops of the hinged joints limited kinematic range, and there was no interaction effect $(p=0.21)$. Stimulation assistance reduced peak forearm loading by $15 \%$ (Table 3 , $p<0.01$ ).

\section{Participant perceptions}

Question themes and general responses about impacts and benefits are presented in Table 4. The participant experienced less discomfort after TKA and resumed standing for tasks that were previously too painful, such as cooking. She appreciated that stimulation improved walking through adequate toe clearance and enhanced endurance. Prior to knee replacement, she was entirely dependent on stimulation for walking as volitional walking was too painful. Stimulation assistance remains her primary method for walking longer distances but she feels less dependent on NMES assistance now. While considering knee replacement surgery, one of her requirements was that the TKA not impact her stimulation system. She made sure the surgeons performing the knee replacements consulted with the research team to avoid disrupting the implanted NMES system and ensure a positive outcome. If she could change the process, she highlighted that she would have gotten the implanted stimulation system immediately after her injury if it was an option. Furthermore, she would prefer the system to have more channels in order to activate additional muscles that might improve balance and thereby reduce reliance on her forearm crutches, or provide redundancy to accommodate for the potential occasional failure of a single electrode.

\section{Discussion}

This is the first known case of an individual with iSCI receiving both an experimental implanted NMES system and a clinically available bilateral TKA. Walking speed and distance decreased as knee pain worsened after prolonged walking with knee hyperextension; knee replacement enabled walking ability that was comparable to gait prior to developing significant knee pain. After knee pain worsened, NMES enabled her to continue walking when she would not have been able to otherwise. The knee replacement significantly reduced pain and limited peak knee hyperextension so she did not need to consciously walk slowly and actively flex her knees. The addition of stimulation improved walking over her best volitional effort both before and after TKA.

That the participant reported value from both interventions suggests it is worth considering integrating various solutions into the comprehensive treatment of persons with complex biomechanical and neuromuscular deficits after iSCI. The key benefit of TKA was reduced pain, while the primary impact of the neuroprosthesis was improved endurance, leg motions, and toe clearance. Despite walking at slower speeds after the TKA than those associated with community ambulation [14], she still reported using the system daily for walking and valued the benefits in endurance, kinematics, and reliability. This highlights the benefit of independence and mobility beyond the impact on gait speed alone. The participant's greatest priority during TKA planning was ensuring that the surgery would not damage the NMES system and remove previously restored function, thus illustrating how highly she valued the implanted stimulation. The subject also identified areas to improve, such as reducing reliance on arm supports beyond the $15 \%$ reduction achieved here (Table 4), which highlights the need to develop more sophisticated control systems for balance and gait stability.

Prior to TKA, knee pain prohibited her from walking without stimulation so she primarily relied on her NMES system for 
walking. The stimulation system facilitated independence and enabled walking in the community. The addition of the knee replacement allowed her to walk without pain again and improved knee mechanics, thus reducing her reliance on stimulation and giving her the option to leave the house without her NMES controller. The improvements in walking ability due to the neuroprosthesis are significant enough that she chooses to complete about half of her walking during the day with stimulation. The value of NMES assistance is especially noticeable and appreciated as she fatigues throughout the day and her voluntary strength decreases.

Since this case report was not planned prior to the knee replacement surgery, an optimal data set was not collected. Ideally, it would have been preferable to have attempted the 6MWT and maximum walk tests with stimulation assistance after knee pain worsened prior to TKA surgery to further isolate the effect of knee replacement. However, the pain and fatigue limited the number of test sessions for the more time intensive assessments and obviated statistical comparisons for some outcome measures. The stimulation pattern was also adjusted to the participant's comfortable walking speed and she was tested without opportunity to practice walking with the modified pattern. Additional practice with the neuroprosthesis may improve her ability to coordinate voluntary effort with stimulation, and allow for faster gait speeds. In spite of the possibility of not fully accommodating to changes in the stimulation patterns, NMES assistance still improved walking performance.

The potential impact of NMES assistance on walking may also have been underrepresented due to the two nonfunctional electrodes. It is reasonable to expect that the additional recruitment of sartorius for hip and knee flexion could have improved swing speed and endurance. Similarly, SHBF might have enhanced damping during loading response, which could extend effective use of that knee joint and enhance kinematics and gait efficiency. There are no current plans to replace the broken lead wires since the walking achievable with the system is sufficient and acceptable to the subject, and the risks involved with a revision surgery are not worth the potential incremental benefits. Should the recruitment properties of the electrodes begin to limit walking ability or increase pain, the malfunctioning electrodes will be disconnected and replaced in a revision surgery to reoptimize the stimulated responses and maximize ambulatory function $[15,16]$.

It is important to note that had her pain worsened several years earlier, she would probably not have been considered a good candidate for the implanted neuroprosthesis. The ability of NMES to restore walking to levels achieved prior to developing knee pain after bilateral TKA suggests there may be preparatory interventions to correct orthopedic or neuromuscular deficits to allow individuals with iSCI to take full advantage of stimulation and maximally improve walking 
ability prior to stimulator implantation. However, interventions like TKA after SCI are still relatively rare. Few if any large and sufficiently powered outcome studies exist to generalize their applicability in the population. Suitability for a TKA and the specific device design will depend on the specific needs and physical characteristics of the recipient based on standards of care and are a matter of clinical judgment [17]. The osteopenia common after SCI could increase the likelihood of TKA failure such as component loosening at the bone/metal interface, which would require surgical revision, and should certainly be considered prior to joint replacement surgery. Furthermore, if stimulation does not correct the imbalances that required the initial surgery, the same issues could arise again. Nevertheless, the complementary combination of TKA and NMES in this single-subject case study successfully addressed different clinical issues to effectively improve ambulatory function beyond what was possible by either intervention alone.

This single-subject study demonstrates the initial impact of a clinical bilateral knee replacement and an experimental implanted neuroprosthesis on walking in a person with iSCI. Although the initial impacts observed in this case are promising, further study is needed to determine the longterm effects in this subject, and to define the indications and clinical characteristics of potential recipients of the combined intervention to ensure optimal clinical outcomes in other individuals with iSCI.

\section{Data availability}

The datasets generated and analyzed during the current study are available from the corresponding author on reasonable request.

Acknowledgements The authors would like to thank Jennifer Kerbo for creating Fig. 1 and editing the videos.

Funding This work was supported by R01NS040547 and KL2TR002547 from the National Institutes of Health as well as 2 I50 RX001871-06, B7692R, and Dr. Triolo's Paul B. Magnusson award from the United States Department of Veterans Affairs Rehabilitation Research and Development Service.

\section{Compliance with ethical standards}

Conflict of interest The authors declare that they have no conflict of interest.

Publisher's note Springer Nature remains neutral with regard to jurisdictional claims in published maps and institutional affiliations.

\section{References}

1. National Spinal Cord Injury Statistical Center, Facts and Figures at a Glance. Birmingham, AL: University of Alabama at Birmingham, 2020.

2. Agarwal S, Kobetic R, Nandurkar S, Marsolais EB. Functional electrical stimulation for walking in paraplegia: 17-year follow-up of 2 cases. J Spinal Cord Med. 2003;26:86-91.

3. Dutta A, Kobetic R, Triolo RJ. Ambulation after incomplete spinal cord injury with EMG-triggered functional electrical stimulation. IEEE Trans Biomed Eng. 2008;55:791-4.

4. Hardin E, Kobetic R, Murray L, Corado-Ahmed M, Pinault G, Sakai J, et al. Walking after incomplete spinal cord injury using an implanted FES system: a case report. J Rehabil Res Dev. 2007;44:333-46.

5. Kobetic R, Triolo RJ, Uhlir JP, Bieri C, Wibowo M, Polando G, et al. Implanted functional electrical stimulation system for mobility in paraplegia: a follow-up case report. IEEE Trans Rehabil Eng. 1999;7:390-8.

6. Koubaa S, Ksibi I, Lebib S, Tlili L, Ben Salah FZ, Dziri C, et al. Total knee arthroplasty in a spinal cord-injured patient: a case report. Ann Phys Rehabil Med. 2009;52:588-93.

7. Zietek P, Dobiecki K. Total knee arthroplasty in patient with paraplegia after spinal cord injury. Acta Chir Orthop Traumatol Cechoslov. 2015;82:84-8.

8. Lombardo LM, Kobetic R, Pinault G, Foglyano KM, Bailey SN, Selkirk S, et al. Impact of an implanted neuroprosthesis on community ambulation in incomplete SCI. J Spinal Cord Med. 2018;41:165-73.

9. Smith B, Peckham PH, Keith MW, Roscoe DD. An externally powered, multichannel, implantable stimulator for versatile control of paralyzed muscle. IEEE Trans Biomed Eng. 1987;34:499-508.

10. Memberg WD, Peckham H, Keith MW. A surgically-implanted intramuscular electrode for an implantable neuromuscular stimulation system. IEEE Trans Rehabil Eng. 1994;2:80-91.

11. Foglyano KM, Schnellenberger JR, Kobetic R, Lombardo L, Pinault G, Selkirk S, et al. Accelerometer-based step initiation control for gait-assist neuroprostheses. J Rehabil Res Dev. 2016;53:919-32.

12. Pasquier G, Ehlinger M, Mainard D. The role of rotating hinge implants in revision total knee arthroplasty. EFORT Open Rev. 2019;4:269-78.

13. Kadaba MP, Ramakrishnan HK, Wootten ME. Measurement of lower extremity kinematics during level walking. J Orthop Res. 1990;8:383-92.

14. Middleton A, Fulk GD, Beets MW, Herter TM, Fritz SL. Selfselected walking speed is predictive of daily ambulatory activity in older adults. J Aging Phys Act. 2016;24:214-22.

15. Delianides C, Tyler D, Pinault G, Ansari R, Triolo R. Implanted high density cuff electrodes functionally activate human tibial and peroneal motor units without chronic detriment to peripheral nerve health. Neuromodulation. 2020;23:754-62.

16. Kilgore KL, Hoyen HA, Bryden AM, Hart RL, Keith MW, Peckham PH. An implanted upper-extremity neuroprosthesis using myoelectric control. J Hand Surg Am. 2008;33: 539-50.

17. Vasso M, Beaufils P, Schiavone Panni A. Constraint choice in revision knee arthroplasty. Int Orthop. 2013;37:1279-84. 